\title{
Ethinsensor für die Online - Transformatorgasanalyse
}

\author{
U. Sasum ${ }^{1}$, T. Widmer ${ }^{1}$, U. Guth ${ }^{2}$ \\ ${ }^{1}$ Forschungszentrum Sensorik Greifswald e.V., Brandteichstrasse 19, 17489 Greifswald, D \\ verein@fsg-ev.de \\ ${ }^{2}$ TU Dresden, FB Chemie und Lebensmittelchemie, Dresden/D
}

\begin{abstract}
Kurzfassung:
Sowohl bei der natürlichen Alterung von Isolierstoffen als auch bei fehlerhaftem Betrieb von mineralölgefüllten elektrischen Betriebsmitteln können sich Gase bilden. Die Kenntnis der Ethinkonzentration ist notwendig, um Rückschlüsse über das Vorhandensein eines Fehlers im Transformator zu ziehen und seine Art zu ermitteln. Es wurde ein neuer NDIR-Ethinsensor für den Einsatz in modularen Onlineanalysatoren für Transformatorgase mit einem Messbereich von 5 ppm bis 10.000 ppm entwickelt und acht Monate an einem Technikumsprüfstand getestet.
\end{abstract}

Key words: Ethin, Transformatorgas, Gas-in-Öl-Analyse, Onlinemessung, Transformatorfehler

\section{Diagnose von Transformatorfehlern}

Sowohl bei der natürlichen Alterung von Isolierstoffen als auch bei fehlerhaftem Betrieb von ölgefüllten Transformatoren und anderen Hochspannungsanlagen können sich Gase bilden. Die Mitte der 1970er Jahre eingeführte Gas-in-Öl-Analyse (DGA) ist heute eines der wichtigsten Verfahren zur Zustandsanalyse dieser Geräte.

Die Fehlergase entstehen im Transformator nicht willkürlich und einzeln. Ihre Bildung wird bestimmt durch die auf das Öl einwirkende Energie. Teilentladungen mit niedriger Energie bewirken hauptsächlich die Bildung von Wasserstoff und in geringerem Maße Methanund Ethanbildung. Elektrische Entladungen (Lichtbogen, Funkenentladung) führen zur Bildung von Wasserstoff und Ethin sowie geringeren Anteilen Methan und Ethen. Thermische Belastungen verursachen im Bereich zwischen $300^{\circ} \mathrm{C}$ und $700^{\circ} \mathrm{C}$ die Entstehung von Ethen und Propen, Wasserstoff und Methan. Dagegen bildet sich bei Temperaturen oberhalb von $700^{\circ} \mathrm{C}$ hauptsächlich Ethen und Propen, auch Wasserstoff. Ist die Temperatur des Heisspunktes größer $1000^{\circ} \mathrm{C}$ entsteht auch Ethin. Die Zelluloseoxidation verursacht die Bildung von grösseren Mengen Kohlenmonoxid und Kohlendioxid.

Durch den Vergleich von DGA-Ergebnissen und den durch die visuelle Inspektion festgestellten Fehlern hat man für die sechs Fehlerarten (Teilentladungen PD, Entladungen niedriger Energie D1, Entladungen hoher Energie D2, thermischer Fehler $\mathrm{t}<300^{\circ} \mathrm{C} \mathrm{T} 1$, thermischer Fehler $300^{\circ} \mathrm{C}<\mathrm{t}<700^{\circ} \mathrm{C}$ T2, thermischer Fehler $\mathrm{t}>700^{\circ} \mathrm{C}$ T3) charakteristische Fehlergasmuster ermittelt.

Sowohl die DIN EN 60599 [1] als auch die Diagnosehilfsmittel bekannter Autoren (MSS [2], Dörnenburg [3], Rogers [4], Duval [5, 6]) nutzen Quotienten bestimmter Fehlergase zur Interpretation der Ergebnisse. Problematisch ist die Diagnose dann, wenn die Kombinationen der Gasquotienten keinem charakteristischen Fehler entsprechen. Es kann sich in diesen Fällen um eine Mischung von Fehlern handeln oder um einen neuen Fehler, der einen alten oder die normale Alterung überlagert.

In einem fehlerfreien luftatmenden Transformator hat sich nach einer gewissen Betriebsdauer im Öl eine Sauerstoffkonzentration von ca. 32000 ppm und eine Stickstoffkonzentration von ca. 64000 ppm eingestellt. Kohlenmonoxid und Kohlendioxid sind ebenfalls im Öl enthalten, Wasserstoff und die C1- bis C3-Kohlenwasserstoffe dagegen nicht. In Tabelle 1 sind die Ergebnisse von Gasin-Öl-Analysen von vier verschiedenen Transformatoren dargestellt. Aus diesen Ergebnissen allein kann nicht festgestellt werden, ob im Transformator ein aktiver Fehler vorhanden ist. Dafür sind in bestimmten zeitlichen Abständen Wiederholungsanalysen nötig, um die durch den Fehler hervorgerufenen Konzentrationsänderungen zu erfassen und zu interpretieren. 
Tab. 1: Ergebnisse von Gas-in-Öl-Analysen verschiedener Transformatoren; Gaskonzentrationen in $\mathrm{ppm}$

\begin{tabular}{|c|c|c|c|c|}
\hline Trafonr.: & 1 & 2 & 3 & 4 \\
\hline $\mathrm{H}_{2}$ & 151 & 157 & 26 & 528 \\
\hline $\mathrm{O}_{2}$ & 20293 & 29664 & 25269 & $<1000$ \\
\hline $\mathrm{N}_{2}$ & 62799 & 64667 & 66305 & 73316 \\
\hline $\mathrm{CO}$ & 60 & 55 & 139 & 997 \\
\hline $\mathrm{CO}_{2}$ & 2384 & 1162 & 4497 & 8775 \\
\hline $\mathrm{CH}_{4}$ & 12 & 538 & 228 & 239 \\
\hline $\mathrm{C}_{2} \mathrm{H}_{6}$ & 5 & 307 & 402 & 251 \\
\hline $\mathrm{C}_{2} \mathrm{H}_{4}$ & 14 & 2548 & 1845 & 44 \\
\hline $\mathrm{C}_{2} \mathrm{H}_{2}$ & 70 & 76 & 7 & $<1$ \\
\hline $\mathrm{C}_{3} \mathrm{H}_{8}$ & 2 & 278 & 373 & 582 \\
\hline $\mathrm{C}_{3} \mathrm{H}_{6}$ & 6 & 3054 & 5224 & 176 \\
\hline
\end{tabular}

\section{Messung von Transformatorgasen}

Die Messmethoden für entsprechende OnlineAnalysatoren und die Messbedingungen der gesamten Messeinrichtung orientierten sich lange Zeit an Standards der klassischen Laboranalytik. Online-Analysatoren bestehen aus einer Ölzuleitung, einer Extraktionseinrichtung und der nachgeschalteten eigentlichen Analysentechnik. Das sind häufig Gaschromatographen. $\mathrm{Da}$ die Gaschromatographie sehr kostenintensiv ist, wurden auch modulare Systeme mit Einzelsensoren gebaut, für die ein robuster, langzeitstabiler Ethinsensor entwickelt werden musste. Die besondere Herausforderung für die Sensorentwicklung bestand darin, dass auch kleine Ethinkonzentrationen unter 20 ppm im Öl neben sehr viel höheren Konzentrationen der C1- bis C3-Kohlenwasserstoffe gemessen werden müssen. Neben dem zu realisierenden Messbereich von 5 ppm bis 10.000 ppm war ein maximales Messküvettenvolumen von $16 \mathrm{ml}$ eine weitere Entwicklungsforderung.

Die Messung der Konzentration von einzelnen Kohlenwasserstoffen in einem Gasgemisch mit Hilfe von Infrarotsensoren ist ohne aufwendige Trennungsschritte für die meisten Kohlenwasserstoffe nicht möglich. Eine Ausnahme bildet das Ethin, da sich dessen Hauptschwingungsbande $(\mathrm{C} \equiv \mathrm{C})$ bei einer Wellenzahl von ca. $3300 \mathrm{~cm}^{-1}$ befindet, während die Valenzschwingungen der übrigen Kohlenwasserstoffe ihre Hauptschwingungsbanden bei ca. $3000 \mathrm{~cm}^{-1}$ besitzen. Jedoch überdecken die Flanken der Absorptions- banden einiger C1- bis C3-Kohlenwasserstoffe die Hauptschwingungsbande des Ethins. Auch Wasserdampf absorbiert in diesem Bereich. Daraus resultieren Querempfindlichkeiten zu diesen Gasen, die sich für die C2-/C3Kohlenwasserstoffe durch eine Positivfilterung, bei der die Störkomponenten in hoher Konzentration in den Messweg gegeben werden, entscheidend senken lassen.

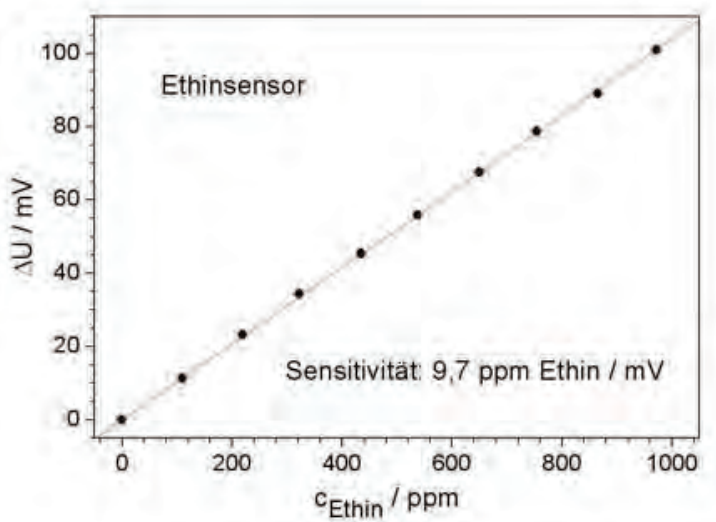

Bild 1. Lineare Empfindlichkeit des Sensors bei kleinen Ethinkonzentrationen

Der Messbereich des Sensors liegt zwischen 5 ppm und 10.000 ppm Ethin. Bis ca. 1.000 ppm zeigt der Sensor einen linearen Anstieg (Signalgrösse/Konzentration). Erst bei höheren Konzentrationen nimmt die Empfindlichkeit allmählich ab. In Bild 1 ist ein Ausschnitt einer Kalibrierkurve dargestellt. Eine Nachkalibrierung nach acht Betriebsmonaten ergab im Vergleich zur Ausgangsmessung nur Abweichungen im Rahmen der Prüfgasgenauigkeit.

Da die Transformatorgasmessung nicht direkt im Öl durchgeführt werden kann, müssen die im Öl gelösten Gase in eine Gasphase überführt werden. Dabei resultieren abhängig von der Extraktionsmethode bei gleicher Konzentration im Öl verschiedene Gasraumkonzentrationen. Hinsichtlich der Sensorkennwerte muss deshalb unterschieden werden, ob sich die Angaben auf die Gaskonzentrationen oder die Konzentrationen der im Öl gelösten Gase unter Einbeziehung des Einflusses der gewählten Extraktionsmethode beziehen. In Tabelle 2 wird die Empfindlichkeit des Sensors gegenüber ausgewählten Transformatorgasen im Verhältnis zum Ethin dargestellt (Konzentration des Gases in ppm für jeweils gleiche Signalgrösse). In der rechten Spalte werden die Empfindlichkeitsverhältnisse bezogen auf im Öl gelöste Gase für eine Gleichgewichtsextraktion mit v(Öl)>>v(Gas) angegeben. 
Tab. 2: Querempfindlichkeiten des Sensors, Gaskonzentrationen in ppm

\begin{tabular}{|c|c|c|c|}
\hline & \multicolumn{3}{|c|}{ Positivfilterung mit } \\
\hline Gas & $100 \% \mathrm{~N}_{2}$ & Transformatorgasgemisch \\
\hline & c im Gas & c im Gas & c im Öl \\
\hline $\mathrm{C}_{2} \mathrm{H}_{2}$ & 1 & 1 & 1 \\
\hline $\mathrm{CH}_{4}$ & 5257 & 5768 & 1928 \\
\hline $\mathrm{C}_{2} \mathrm{H}_{6}$ & 65 & 93 & 205 \\
\hline $\mathrm{C}_{2} \mathrm{H}_{4}$ & 52 & 414 & 580 \\
\hline $\mathrm{C}_{3} \mathrm{H}_{8}$ & 39 & 68 & 510 \\
\hline $\mathrm{C}_{3} \mathrm{H}_{6}$ & 137 & 271 & 1860 \\
\hline
\end{tabular}

Trotz sehr geringer Wasserkonzentrationen (Grenzwert: < $25 \mathrm{mg} / \mathrm{kg}$ ) im Betriebsöl von Transformatoren, können je nach Extraktionsmethode im Messgas mehrere Tausend Vol-ppm Wasserdampf vorhanden sein. Diese Querempfindlichkeit kann nicht durch Positivfilterung verringert, aber aus Feuchtemessungen gut verrechnet werden. Im Gas verursacht eine Wasserkonzentration von $1500 \mathrm{ppm}$ ein Ethinsignal von ca. $13 \mathrm{ppm}$. Zwischen 1500 ppm und 20000 ppm entspricht die Querempfindlichkeit je 1000 ppm Wasser einer Ethinkonzentration von 4,2 ppm.

\section{Online-Messungen}

Um die Leistungsfähigkeit des neuen Ethinsensors (Bild 1) unter Betriebsbedingungen in einem Onlineanalysator prüfen zu können, wurde dieser in einen Transformatorgasmonitor (TGM, Gatron $\mathrm{GmbH}$ ) eingebaut (Bild 2). Der TGM wurde an ein Technikumsölsystem angeschlossen. In diesem System können die Konzentrationen der im Öl gelösten Gase in einem grossen Bereich variiert werden. Die Entnahme von repräsentativen Ölproben für Gas-in-ÖlAnalysen ist problemlos möglich. Begleitend zur Onlinemessung mit dem Ethinsensor wurden regelmäßig Gas-in-Öl-Analysen durchgeführt.

Eine Onlinemessung stellt an Sensoren durch wechselnde Temperatur- und Luftdruckverhältnisse sowie möglicherweise auftretende Langzeitdriften besondere Anforderungen. Diese Faktoren wirken sich weniger auf die Empfindlichkeit des Sensors, als auf seinen Nullpunktswert aus. Um die genannten Einflüsse zu minimieren, wurde vor der eigentlichen Messung des extrahierten Transformatorgases der Sensor mit Umgebungsluft kalibriert und der so gewonnene
Nullpunktswert zur Konzentrationsberechnung genutzt. Bedingt durch dieses Messregime nahmen die Konzentrationen der dem ÖI zugesetzten, gelösten Gase stetig ab.

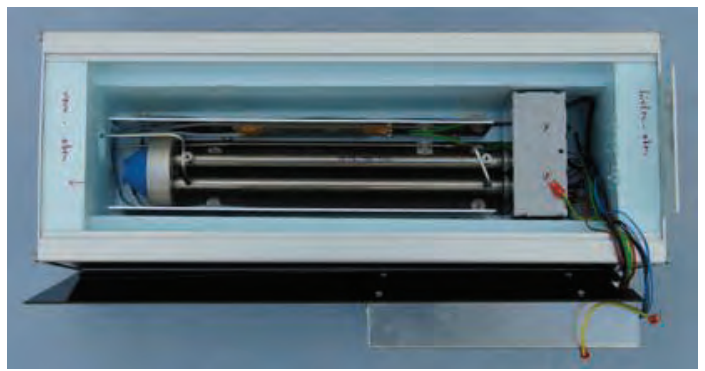

Bild 1 Ethinsensor, Gehäuse geöffnet

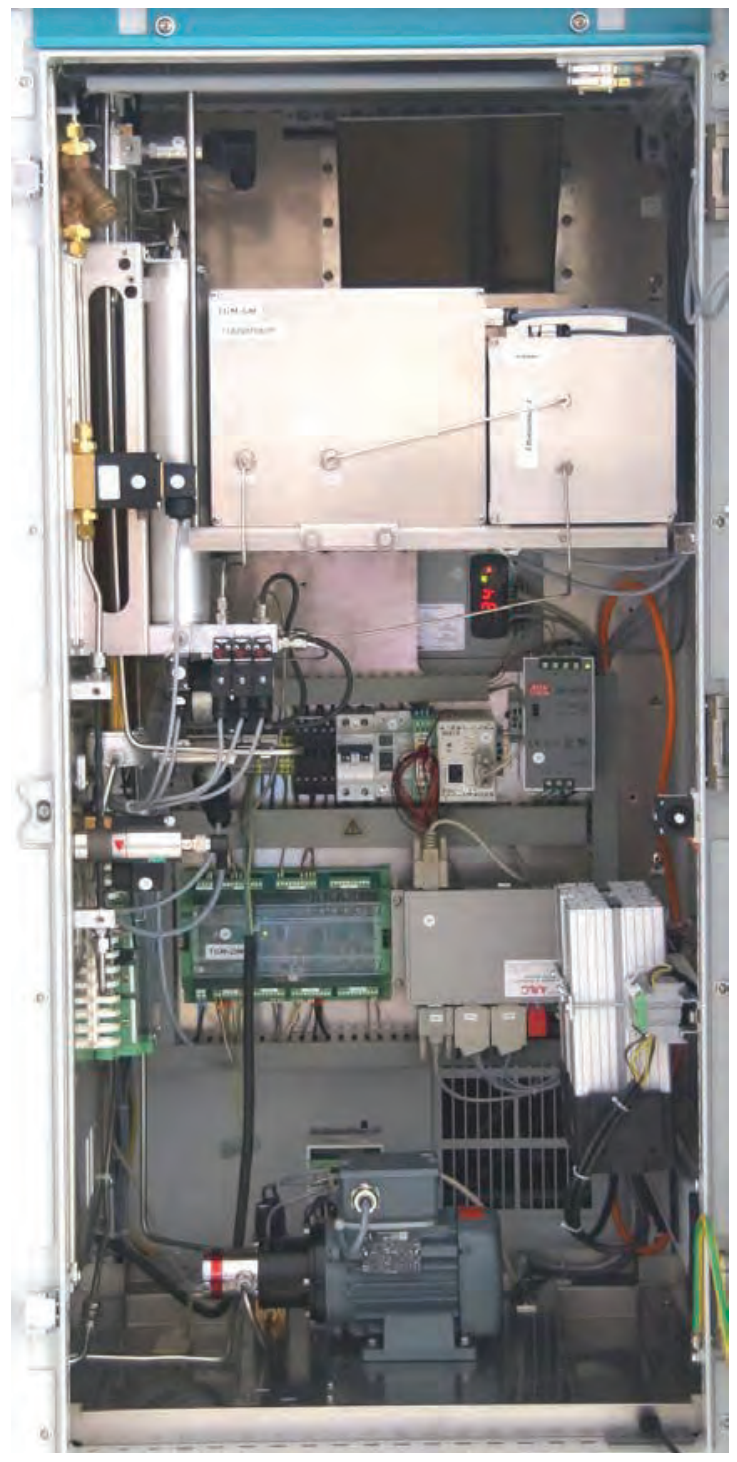

Bild 2 Etinsensor in einem Transformatorgasmonitor TGM (Gatron $\mathrm{GmbH}$ ) 


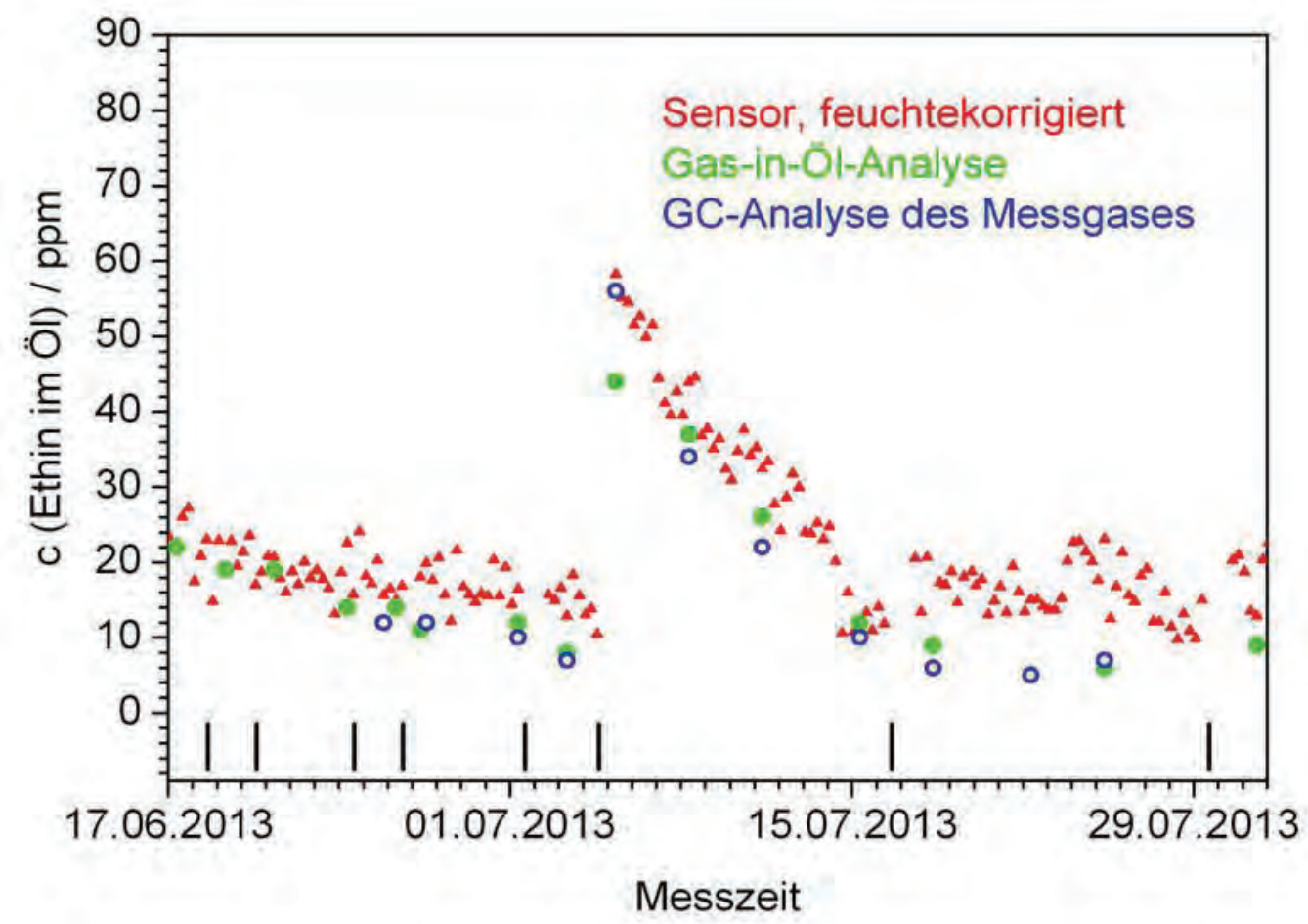

Bild 3 Onlinemessung am Technikumsprüfstand; die schwarzen Striche markieren die Zeitpunkte von Gaszugaben in das Betriebsöl (siehe Tab. 4)

Tab. 3: Ergebnisse von Gas-in-Öl-Analysen des Technikumsprüfstandes, Konzentrationen in ppm

\begin{tabular}{|c|c|c|c|c|c|c|}
\hline & $\mathrm{CH}_{4}$ & $\mathrm{C}_{2} \mathrm{H}_{6}$ & $\mathrm{C}_{2} \mathrm{H}_{4}$ & $\mathrm{C}_{2} \mathrm{H}_{2}$ & $\mathrm{C}_{3} \mathrm{H}_{8}$ & $\mathrm{C}_{3} \mathrm{H}_{6}$ \\
\hline 17.06 .13 & 2 & 178 & 78 & 22 & 48 & 22 \\
\hline 19.06.13 & 1032 & 170 & 73 & 19 & 51 & 24 \\
\hline 21.06.13 & 629 & 299 & 72 & 19 & 45 & 20 \\
\hline 26.06 .13 & 1139 & 282 & 65 & 14 & 45 & 18 \\
\hline 27.06.13 & 992 & 1256 & 62 & 11 & 69 & 68 \\
\hline 01.07.13 & 775 & 1145 & 60 & 12 & 66 & 56 \\
\hline 03.07 .13 & 708 & 1073 & 1526 & 8 & 80 & 39 \\
\hline 05.07 .13 & 1924 & 977 & 1316 & 44 & 44 & 16 \\
\hline 08.07 .13 & 1088 & 991 & 1158 & 37 & 40 & 27 \\
\hline 10.07.13 & 915 & 1032 & 1191 & 26 & 55 & 26 \\
\hline 15.07 .13 & 822 & 817 & 825 & 12 & 55 & 26 \\
\hline 18.07.13 & 1472 & 1555 & 1309 & 9 & 1027 & 605 \\
\hline 22.07 .13 & 1303 & 1423 & 1114 & 5 & 1012 & 595 \\
\hline 25.07 .13 & 1065 & 1100 & 829 & 6 & 780 & 446 \\
\hline 31.07 .13 & 1141 & 1572 & 1211 & 9 & 971 & 551 \\
\hline
\end{tabular}


In Bild 3 sind Ergebnisse der Onlinemessungen dargestellt. In diesem Zeitraum wurde die Konzentration der Kohlenwasserstoffe im Öl mehrfach durch Gaszugabe verändert.

Tab. 4: Änderung der Konzentrationen der im ÖI gelösten Gase durch Gaszugabe in das Ölsystem

\begin{tabular}{|c|c|}
\hline Datum der Gaszugabe & Zugegebenes Gas \\
\hline 18.06 .13 & Methan \\
\hline 20.06 .13 & Ethan \\
\hline 24.06 .13 & Methan \\
& Ethan \\
\hline 26.06 .13 & Ethan \\
\hline 01.07 .13 & Ethen \\
\hline 04.07 .13 & Ethin \\
& Methan \\
\hline 16.07 .13 & Methan \\
& Ethan \\
& Ethen \\
& Propan \\
& Propen \\
\hline 29.07 .13 & Ethin \\
& Methan \\
& Ethan \\
& Ethen \\
\hline
\end{tabular}

Die Überprüfung der Richtigkeit der Messergebnisse erfolgte nicht nur durch Gasin-Öl-Analysen (Tab. 3). Unmittelbar nach ausgewählten Messungen wurde Messgas aus dem Gasraum des Onlinesensors entnommen und gaschromatographisch analysiert. Mit einer Ausnahme stimmen diese Ergebnisse gut überein. Nur am 05.07.13 wichen die Ergebnisse der DGA einerseits und der Sensorbzw. GC-Messungen andererseits deutlich voneinander ab. Fehleranalysen ergaben, dass das Verteilungsgleichgewicht des Ethins zwischen dem Gasraum des Onlineanalysators und dem Ölsystem nach der Gaszugabe am 04.07.13 noch nicht eingestellt war.

In Bild 4 wird der Einfluss der Feuchte im Messgas deutlich. Das sehr feuchte Transformatoröl im Technikumsprüfstand (ca. $25 \%$ relative Ölfeuchte bei $32^{\circ} \mathrm{C}$ ) wurde im Bypass getrocknet. Das eingesetzte Trockenmittel adsorbiert die C1- bis C3Kohlenwasserstoffe nicht. Der Trocknungsvorgang wurde durch Messung der relativen Ölfeuchte mit einem Sensor MMT318 (Vaisala) verfolgt.

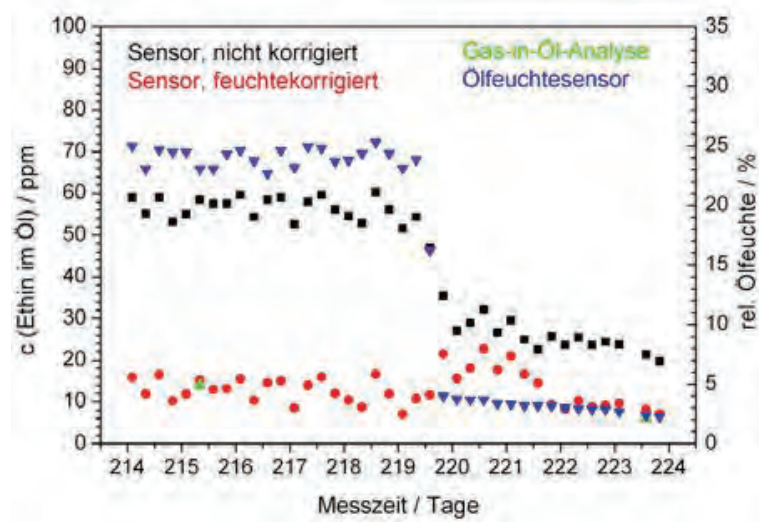

Bild 4. Feuchtequerempfindlichkeit des Ethinsensors

Nach der rechnerischen Feuchtekorrektur der gemessenen Ethinkonzentrationen stimmen die Ergebnisse der Onlinemessungen und der Gasin-Öl-Analysen (Tab. 5) gut überein. Der Anteil der anderen Kohlenwasserstoffe am Ergebnis der Onlinemessung beträgt sowohl am Tag 215 als auch am Tag 225 max. 4 ppm Ethin im Öl.

Tab. 5: Kohlenwasserstoffkonzentrationen in ppm im Öl, Ergebnisse der Gas-in-Öl-Analysen

\begin{tabular}{|c|c|c|}
\hline & Tag 215 & Tag 225 \\
\hline $\mathrm{CH}_{4}$ & 323 & 307 \\
\hline $\mathrm{C}_{2} \mathrm{H}_{6}$ & 576 & 636 \\
\hline $\mathrm{C}_{2} \mathrm{H}_{4}$ & 413 & 429 \\
\hline $\mathrm{C}_{2} \mathrm{H}_{2}$ & 14 & 6 \\
\hline $\mathrm{C}_{3} \mathrm{H}_{8}$ & 387 & 466 \\
\hline $\mathrm{C}_{3} \mathrm{H}_{6}$ & 203 & 241 \\
\hline
\end{tabular}

\section{Zusammenfassung}

Es wurde ein wartungsarmer und kostengünstiger NDIR-Sensor zur Ethinmessung in Transformatorgasen mit einem Messbereich zwischen $5 \mathrm{ppm}$ und 10.000 ppm entwickelt. Im Bereich bis ca. 1.000 ppm zeichnet sich der Sensor durch eine lineare Empfindlichkeit aus. Die aufgrund der Charakteristik der IR-Spektren vorhandenen Querempfindlichkeiten des Sensors für die anderen, in Transformatorgasen potentiell in grossem Überschuss enthaltenen Kohlenwasserstoffe konnten durch eine zusätzliche Positivfilterung erheblich verringert werden. Die Querempfindlichkeit gegenüber Wasserdampf kann dadurch nicht verringert, aber mit Hilfe von Feuchtemessungen gut verrechnet werden. Nach Einbau des Senors in einen 
Transformatorgasmonitor TGM an einem Technikumsprüfstand wurden über einen Zeitraum von acht Monaten die Ethinkonzentrationen im Öl ermittelt. Die Ergebnisse der Ethinmessungen mit dem Sensor und begleitender Gas-in-Öl-Analysen stimmten gut überein.

\section{Literatur}

[1] DIN EN 60599 (VDE 0370-7):2008-02, In Betrieb befindliche, mit Mineralöl impregnierte elektrische Geräte - Leitfaden zur Interpretation der Analyse gelöster und freier Gase

[2] Müller, R.; Schliesing, H.; Soldner, K., Die Beurteilung des Betriebszustandes von Transformatoren durch Gasanalyse, Elektrizitätswirtschaft 76 (11), 345-9 (1977

[3] Dörnenburg, E., Strittmatter, W., Überwachung von Öltransformatoren durch Gasanalyse, Brown Boveri Mitt. 1974 (5) 238-47

[4] Rogers, R. R.; IEEE and IEC codes to internal incipient faults in transformers using gas in oil analysis 13, (59) 349-54 (1978), IEEE Trans. Elektr. Insulation 13 (5), 349-54 (1978)

[5] Duval, M., Fault gases formed in oil-filled breathing EHV power transformers - The interpretation of gas analysis data, IEEE PAS Conf. Paper No C 74 476-8, 1974

[6] Duval, M., Gervais, P., Belanger, G., Update on Hydro-Quebec's Experience in the Interpretation of Dissolved Gas Analysis in HV Transformers, CIGRE Symposium 1993, Berlin, Paper 110-14 